\title{
miR-324-3p suppresses migration and invasion by targeting WNT2B in nasopharyngeal carcinoma
}

\author{
Chao Liu ${ }^{1,2}$, Guo Li ${ }^{1,2}$, Nianting Yang ${ }^{1,2}$, Zhongwu Su ${ }^{1,2}$, Shuiting Zhang ${ }^{1,2}$, Tengbo Deng ${ }^{1,2}$, Shuling Ren ${ }^{1,2}$, \\ Shanhong Lu ${ }^{1,2}$, Yongquan Tian ${ }^{1,2}$, Yong Liu ${ }^{1,2^{*}+}$ and Yuanzheng Qiu ${ }^{1,2^{*}+}$
}

\begin{abstract}
Background: Nasopharyngeal carcinoma (NPC) is a malignant epithelial carcinoma of the head and neck with strong ability of invasion and metastasis. Our previous study indicated that miR-324-3p, as a tumor-suppressive factor, could regulate radioresistance of NPC cells by targeting WNT2B. The purpose of this study is to investigate the role of miR324-3p on migration and invasion in NPC cells.

Methods: Quantitative real time PCR was applied to measure the expression level of miR-324-3p and WNT2B mRNA in both cells and tissues, and the expression level of WNT2B protein was determined by western blotting. The capacity of migration and invasion were tested by using wound healing and transwell invasion assay.

Results: Ectopic expression of miR-324-3p or silencing its target gene WNT2B could dramatically suppress migration and invasion capacity of NPC cells. Meanwhile, the alterations of miR-324-3p in NPC cells could influence the expression level of the biomarkers of epithelial-mesenchymal transition (EMT), including E-cadherin and Vimentin. Moreover, the expression of miR-324-3p was obviously downregulated and WNT2B was significantly upregulated in NPC tissues. The expression levels of miR-324-3p and WNT2B were closely correlated with T stage, clinic stage and cervical lymph node metastasis of NPC $(P<0.05)$.
\end{abstract}

Conclusion: miR-324-3p could suppress the migration and invasion of NPC by targeting WNT2B and the miR-324-3p/ WNT2B pathway possibly provide new potential therapeutic clues for NPC.

Keywords: Nasopharyngeal carcinoma, miR-324-3p, WNT2B, Invasion

\section{Background}

Nasopharyngeal carcinoma (NPC) is an Epstein-Barr virus associated cancer that mostly occurs in Southern China and South-Eastern Asia [1]. Despite great improvements in chemoradiotherapy over the past few decades, the overall 5-year survival rate for NPC still remains poor [2]. Strong ability to migrate and invade is a leading cause for the dismay prognosis of advanced NPC patients [3]. For this reason, exploring the molecular mechanisms

\footnotetext{
*Correspondence: liuyongent@csu.edu.cn; xyqyz@hotmail.com

†Yong Liu and Yuanzheng Qiu are the co-corresponding authors

${ }^{1}$ Department of Otolaryngology Head and Neck Surgery, Xiangya Hospital, Central South University, 87 Xiangya Road, Changsha 410008, Hunan, China

Full list of author information is available at the end of the article
}

underlying NPC migration and invasion is essential for the development of novel therapeutic strategies.

microRNAs (miRNAs) are a kind of small non-protein-coding RNA, which consist of 19-25 nucleotides [4]. They can inhibit target gene expression at the posttranscriptional level by pairing with the $3^{\prime}$ untranslated regions ( $3^{\prime}$ UTRs), $5^{\prime}$ UTRs or coding region of mRNAs $[5,6]$. Accumulated evidences prove that dysregulated miRNAs can function as oncogenes or tumour suppressors in the initiation and progression of various human cancers [7]. In NPC, miRNAs are also reported to play important regulatory roles in many critical biological processes including cell proliferation, apoptosis, radiosensitivity and chemosensitivity [8-10]. As to migration and invasion of NPC, a series of miRNAs like miR-145 or 
miR-744 can inhibit or enhance NPC migration and invasion by targeting SMAD3 or ARHGAP5, respectively [11, 12]. These above findings indicated that miRNAs provide a new perspective for the investigation of migration and invasion in NPC.

In our preliminary study, we have found that miR324-3p could regulate the radioresistance of NPC cells and further confirmed WNT2B was the target gene of miR-324-3p [13]. Here, we focused on the roles of miR324-3p involved in NPC migration and invasion. Our results showed that miR-324-3p inhibited migration and invasion of NPC cells and affected epithelial-mesenchymal transition (EMT) biomolecules, and the target gene $W N T 2 B$ could enhance the migration and invasion ability of NPC. Currently, in NPC tissue specimens, miR324-3p was found to be downregulated while WNT2B was upregulated. Moreover, the expression levels of miR324-3p and WNT2B were associated with stages of NPC, as well as with lymph node metastasis. These results provide valuable clues toward understanding the molecular mechanisms of NPC migration and invasion.

\section{Methods}

\section{Cell cultures}

NPC cell lines 5-8F and 6-10B were purchased from the Cell Center of Central South University, Changsha, China. The cells were cultured in RPMI medium 1640 (Hyclone, Logan, UT, USA) with 10\% foetal bovine serum (Gibco BRL, Gaithersburg, MD, USA) and were incubated at $37{ }^{\circ} \mathrm{C}$ in a humidified chamber with $5 \% \mathrm{CO}_{2}$. Cells in an exponential growth state were used for subsequent experiments.

\section{Patients and tissue preparation}

Primary NPC $(\mathrm{n}=39)$ and normal nasopharyngeal epithelium (NPE) $(n=21)$ tissues were obtained from the the Department of Otolaryngology Head and Neck Surgery, Xiangya Hospital, Central South University, Changsha, China. All patients had no history of previous malignancies. Staging was performed by the 2008 NPC staging system of China. The study protocol was approved by the Research Ethics Committee of the Central South University, Changsha, China. Informed consents were obtained from all of the patients.

\section{Oligonucleotides and transfection}

miR-324-3p mimics, WNT2B siRNA and negative control (NC) were chemically synthesized from GenePharma Co., Shanghai, China. The NPC cells 5-8F and 6-10B were transfected with Lipofectamine 2000 reagent (Invitrogen, Burlington, ON, Canada) according to the manufacturer's instructions. The transfection efficiency was observed by fluorescence microscopy, and the expression level of miR-324-3p and WNT2B were evaluated using the qRT-PCR examination System (Bio-Rad, Hercules, CA, USA).

\section{RNA extraction and quantitative real-time PCR (qRT-PCR) analyses}

TRIzol reagent (Invitrogen, Carlsbad, CA, USA) was used to extract the total RNA from NPC cells and tissues. The All-in-One ${ }^{\mathrm{TM}}$ miRNA qRT-PCR Detection Kit (GeneCopoeia Inc., MD, USA) was applied in reverse transcription and quantitative detection of miRNAs according to the user manual. The detection of mRNAs was carried out with TaqMan Reverse Transcription Reagents and SYBR Green PCR Master Mix (Applied Biosystems, CA, USA). PCR quantification was conducted using the $2^{-\Delta \Delta C T}$ method and normalized to U6 for miRNA or GAPDH for mRNA. The sequences of the primers used for the PCR are as follows: WNT2B forward, $5^{\prime}$-TGG CGT GCA CTC TCA GAT TT- $3^{\prime}$ and reverse, $5^{\prime}$-GAC AAG ATC AGT CCG GGT GG-3'; GAPDH forward, 5'-TCC AAA ATC

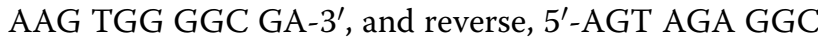
AGG GAT GAT GT- $3^{\prime}$. The technical documentation of qRT-PCR was listed in Additional file 1, and representative data of standard and melt curves of the premirs were listed in Additional file 2: Figure S1. And the validation of the stability of the reference genes between NPC and NPE was showed in Additional file 2: Figure S2.

\section{Wound healing assay}

Wound-healing assay was used to examine the cell migration activity. When the cells were grown to reach almost total confluence (nearly 36-48 h after transfection), a $10 \mu \mathrm{l}$ plastic pipette tip was adopted to creat an artificial wound. Then the cells were cultured in serumfree medium. The initial gap length $(0 \mathrm{~h})$ and the residual gap length $24-72 \mathrm{~h}$ after scratching were observed under the inverted microscope. Experiments were performed in triplicate.

\section{Cell invasion assay}

A transwell invasion assay was performed according to the operating instruction. Briefly, $1 \times 10^{4}$ cells with serum-free medium were seeded to the top chamber with Matrigel-coated membrane (BD Biosciences, Bedford, MA, USA). After a $48 \mathrm{~h}$ incubation period, noninvading cells on the surface of the upper chamber were removed with a cotton swab. Cells on the lower side of the chamber were fixed in paraformaldehyde, stained with crystal violet, and then counted in five random fields under microscope. Each experiment was done three times. 


\section{Western blotting analysis}

Western blotting was carried out as described previously. Total cell or tissue proteins were extracted and separated on $10 \%$ SDS-PAGE gels, and electroblotted onto PVDF (polyvinylidene fluoride) membranes (Millipore, Billerica, MA, USA). Then, the protein expression was detected by incubation with the relevant primary antibody followed by an appropriate secondary antibody. The primary antibody in current study included anti-WNT2B (1:800, Boiss Inc., Woburn, MA, USA), anti-E-cadherin and anti-Vimentin (1:800, Cell Signaling Technology, Danvers, MA, USA). Anti- $\beta$-Actin or antiGAPDH (1:1000, Beyotime, Shanghai, China) were used as the loading control. The relative expression of WNT2B protein was calculated by the image intensity of the ratio of WNT2B and $\beta$-Actin.

\section{Statistical analysis}

All data shown are representative results of at least three independent experiments and were expressed as mean \pm standard deviation (SD). Statistical analyses were performed using a two-sided unpaired Student's $t$ test (for equal variances) or Mann-Whitney $U$ test (for unequal variances) with SPSS 18.0 software. $P<0.05$ was considered statistically significant.

\section{Results}

\section{Ectopic expression of miR-324-3p inhibits migration} and invasion of NPC cells

To investigate the role of miR-324-3p on NPC migration and invasion, we upregulated the expression of miR324-3p by transfecting miR-324-3p mimic into NPC 5-8F and $6-10 \mathrm{~B}$ cells. A transfection efficiency of $93.8 \pm 2.1$ and $92.7 \pm 3.3 \%$ was observed under fluorescence microscopy in 5-8F and 6-10B cells, respectively, and the expression of miR-324-3p was successfully increased $(P<0.01$; Fig. 1a, b). The wound healing assay showed that cell migratory abilities of miR-324-3p overexpressing cells were greatly inhibited compared with uninfected mock and control cells $(P<0.01$, Fig. 1c, d). The transwell invasion assay showed that the number of invading cells was significantly decreased in miR-324-3p upregulated cells $(P<0.01$, Fig. $1 \mathrm{e}, \mathrm{f})$. These results indicated that ectopic expression of miR-324-3p could inhibit migration and invasion of NPC cells.

\section{Silencing expression of WNT2B suppresses migration and invasion of NPC cells}

Our previous study had shown that WNT2B was the target gene of miR-324-3p, so we transfected WNT2B siRNA plasmids into NPC cells to confirm its role on migration and invasion of NPC. The expression of
WNT2B was obviously decreased in 5-8F and $6-10 \mathrm{~B}$ cells by qRT-PCR detection (Fig. 2a, b). The wound healing assay demonstrated that cell migration ability was inhibited when WNT2B was downregulated $(P<0.01$, Fig. 2c, d). The transwell invasion assay showed that the number of invading cells was significantly reduced in WNT2B siRNA transfected cells $(P<0.01$, Fig. 2e, f). These data confirmed that the target gene WNT2B could also affect the migration and invasion of NPC cells.

\section{miR-324-3p alters the expression of EMT biomarkers E-cadherin and Vimentin}

EMT was a classical mechanism of tumour invasion and metastasis, and we had confirmed that WNT2B could affect the expression of EMT biomarkers in previous study [14]. Here, we found following the upregulation of miR-324-3p in both NPC 5-8F and 6-10B cells, the expression of epithelial markers E-cadherin was upregulated while mesenchymal markers Vimentin was downregulated (Fig. 3), which suggested that EMT might participate in the process of miR-324-3p mediated migration and invasion of NPC.

miR-324-3p is downregulated and its target gene WNT2B is upregulated in NPC specimens

We have validated that both miR-324-3p and target gene WNT2B could affect migration and invasion of NPC, so we further quantified the expressions of miR-324-3p and WNT2B in 39 freshly frozen NPC and 21 normal NPE tissues. The results showed that in NPC tissues, the expression of miR-324-3p was significantly decreased and both WNT2B mRNA and protein were upregulated (Fig. 4). And the expression of miR-324-3p was negative correlated with WNT2B mRNA and protein (Additional file 2: Figure S3).

\section{Correlationship between miR-324-3p/WNT2B expression and clinicopathological parameters}

The relationship between miR-324-3p/WNT2B expression and clinicopathological characteristics of NPC was explored. As summarized in Table 1, miR-324-3p lower expression was associated with tumour $\mathrm{T}$ classification, clinic stage and lymph node metastasis $(P<0.05)$, respectively. And WNT2B mRNA/protein overexpression was also associated with tumour $\mathrm{T}$ classification, clinic stage and lymph node metastasis $(P<0.05)$. However, no significant relationship existed between these markers and variables such as gender and age $(P>0.05)$. More expression data of NPE and NPC with two differential stratifications of $\mathrm{T}$ and clinic stage forms was shown in Additional file 2: Figure S4. 


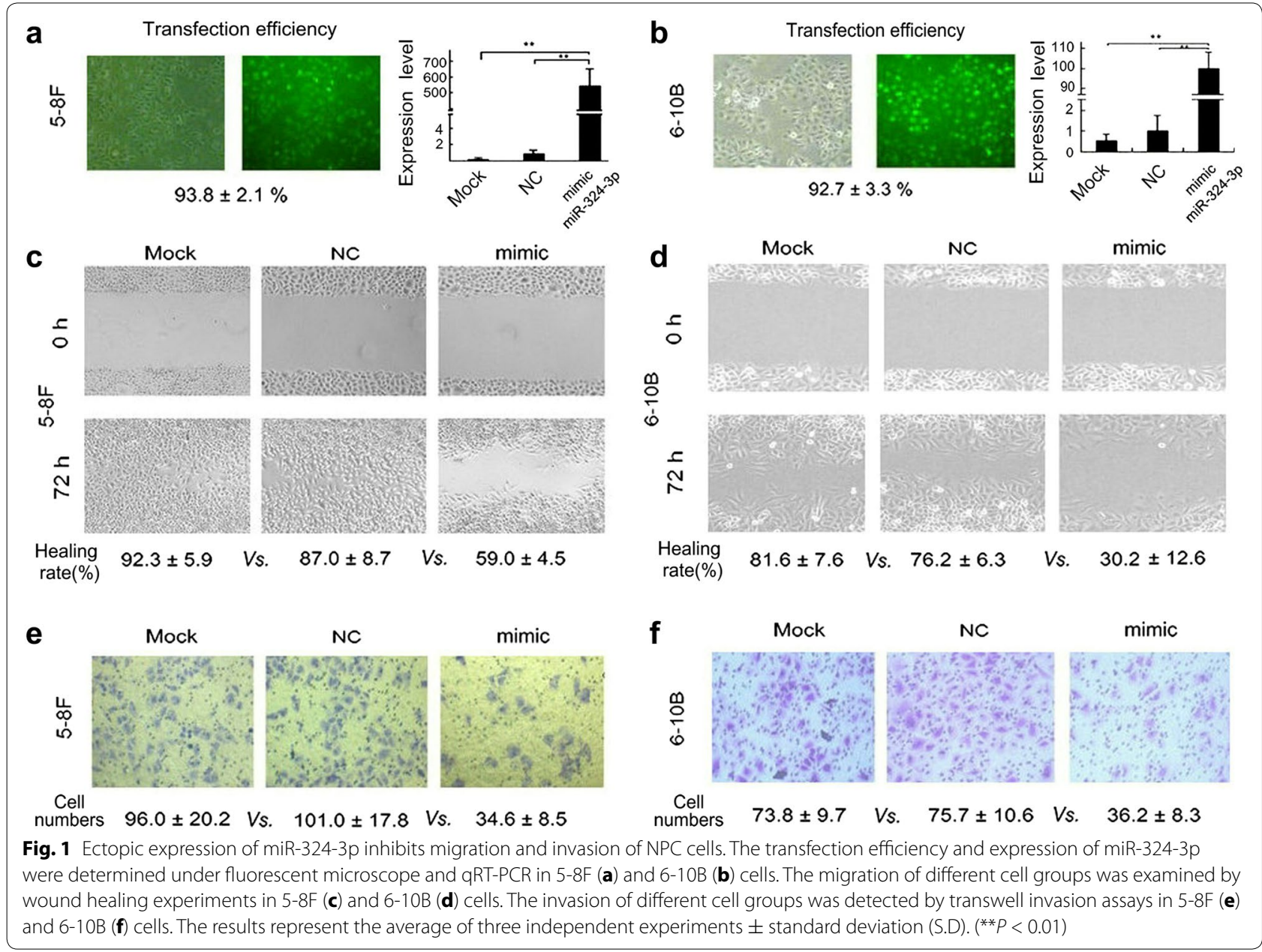

\section{Discussion}

To understand the underlying molecular mechanisms involved in NPC migration and invasion is beneficial to develop better therapeutic strategies for NPC patients. Recently, as important regulatory factors, miRNAs have been shown to play crucial roles in various malignant biobehaviours of tumours, including NPC migration and invasion. Our previous study indicated that miR-324-3p could target WNT2B to affect the radioresistance of NPC in vitro [13]. In this study, we further demonstrated that miR-324-3p could also regulate the migration and invasion of NPC, which contributes to illustrate the complex molecular mechanisms of migration and invasion in NPC.

miR-324-3p was located in the region of human chromosome 17p13.1 and firstly identified in mammalian neurons [15]. Up to now, with the rapid development of microarray and sequencing technology, some researchers have found that miR-324-3p was dysregulated in a variety of tumours such as breast cancer [16], hepatocellular carcinoma [17] and pancreatic cancer [18]. However, none of these studies further explored the roles of miR-324-3p on tumour maglinant biobehaviours. More specifically, previous investigations into the function of miR-324-3p were limited, and only several reports showed miR324-3p was a target of ACE inhibition to promote renal fibrosis and miR-324-3p could target RelA promoter to induce its expression in an Ago2 dependent manner in cells of neural origin $[19,20]$. Functional analyses of miR324-3p on tumour were just seen in our preceding study [13] and another similar report of NPC radioresistance, in which SMAD7 was validated as the target of miR324-3p [21]. In this study, we further confirmed the role of miR-324-3p on NPC migration and invasion, which suggests miR-324-3p as an anti-tumour miRNA.

miRNAs usually exert their function by interacting with their target genes via base pairing. In our previous research, we found that $W N T 2 B$ was a direct target gene of miR-324-3p and confirmed WNT2B could affect radioresistance of NPC cells [13]. WNT2B was known to stimulate the canonical WNT/ $\beta$-catenin pathway and affected various malignant tumour progression [22]. In head and 


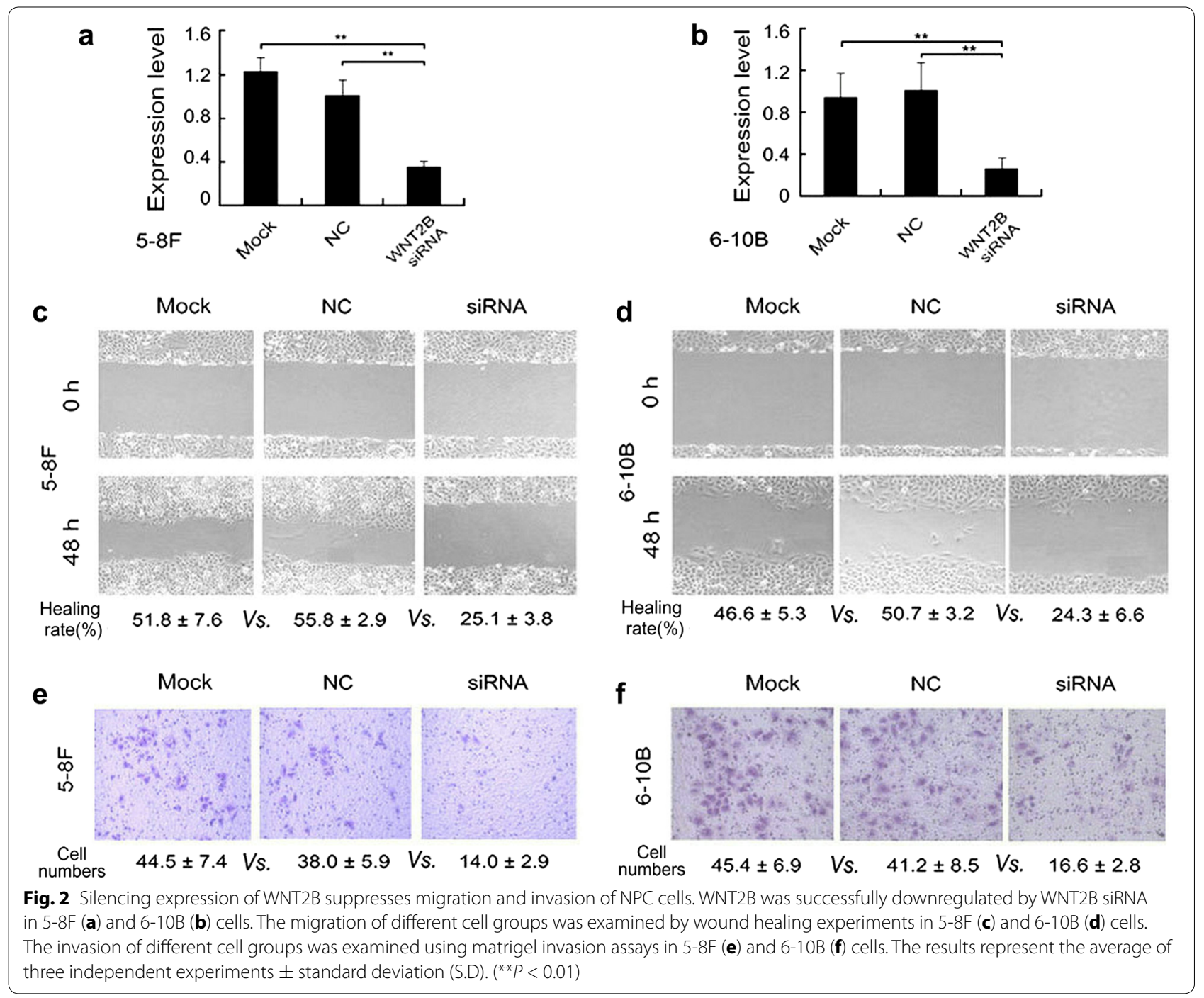

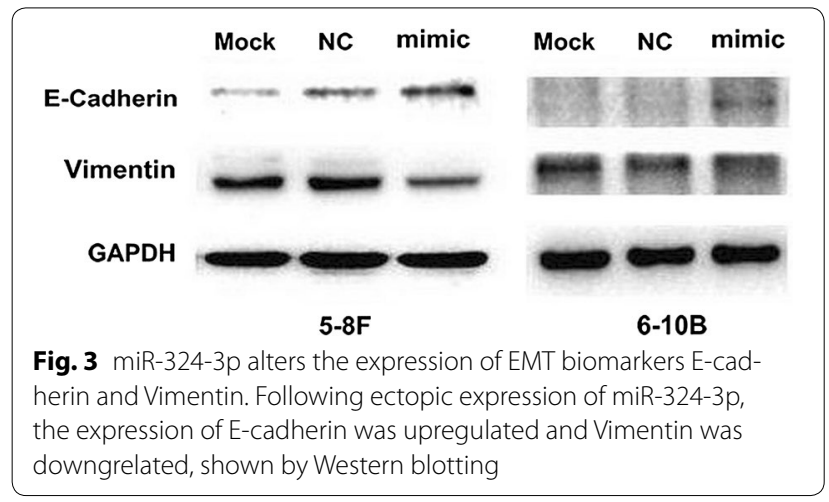

neck squamous cell carcinoma, WNT2B played a role in tumourigenesis and chemotherapy resistance in vivo and in vitro [23]. Intratumoural WNT2B was reported to be correlated with the expression of Survivin and c-Myc, tumour proliferation and prognosis in malignant pleural mesothelioma [24]. Jiang et al. also found that the highexpression level of $W N T 2 B$ was associated with the progression and worse outcome of pancreatic cancer [25]. In addition, $W N T 2 B$ can increase the ability of metastasis and chemoresistance of ovarian cancer through the caspase-9/BCL2/BCL-xL pathway and EMT/p-AKT pathways [26]. All these studies highlighted the role of $W N T 2 B$ on tumour malignant processes. However, the function of WNT2B on NPC was only seen in our preliminary work, and here we further found that $W N T 2 B$ played roles on migration and invasion of NPC, and revealed that both WNT2B mRNA and protein was positively correlated with higher stages of NPC, which indicated the possibility of WNT2B to be the novel biomarker of NPC.

EMT was defined as a process that epithelial cells transformed into a mesenchymal phenotype, in which the expression of epithelial marker E-cadherin and 

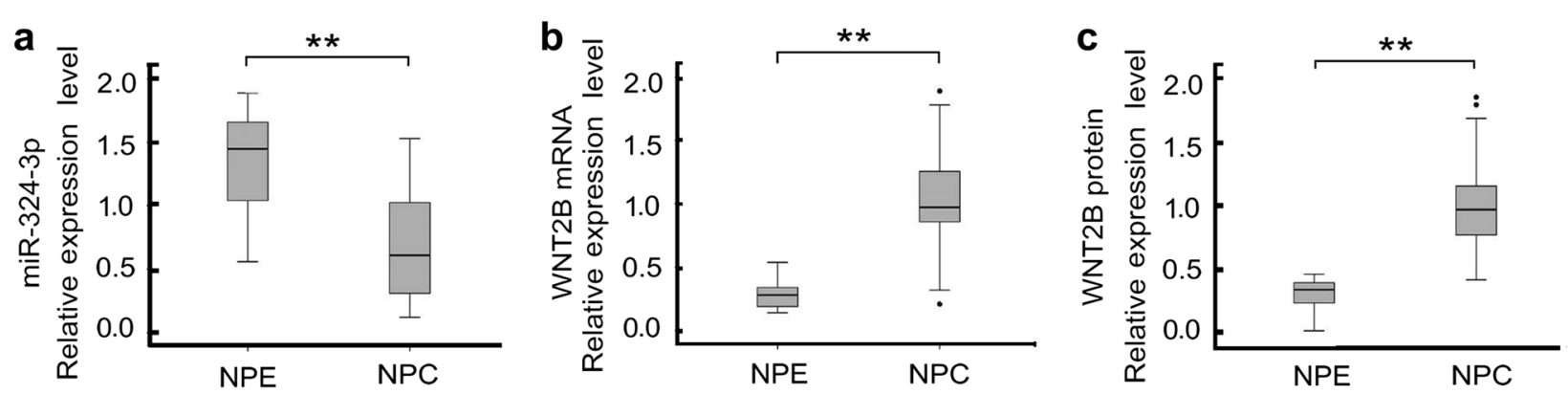

Fig. 4 The expression of miR-324-3p and WNT2B in NPC specimens. a miR-324-3p was downregulated in NPC tissues compared with NPE tissues. b WNT2B mRNA was upregulated in NPC tissues compared with NPE tissues. CWNT2B protein was upregulated in NPC tissues compared with NPE tissues. miR-324-3p, WNT2B mRNA and protein were normalized with the internal control U6, GAPDH and $\beta$-Actin, respectively. ( $\left.{ }^{* *} P<0.01\right)$

Table 1 Clinicopathological features of the cases of NPC

\begin{tabular}{|c|c|c|c|c|}
\hline Variables & Cases & miRNA-324-3p & WNT2B mRNA & WNT2B protein \\
\hline \multicolumn{5}{|l|}{ Sex } \\
\hline Male & 28 & $0.717 \pm 0.463$ & $1.028 \pm 0.461$ & $1.001 \pm 0.369$ \\
\hline Female & 11 & $0.613 \pm 0.245$ & $1.052 \pm 0.305$ & $1.010 \pm 0.320$ \\
\hline \multicolumn{5}{|l|}{ Age } \\
\hline$<45$ & 19 & $0.731 \pm 0.386$ & $1.055 \pm 0.372$ & $1.008 \pm 0.301$ \\
\hline$\geq 45$ & 20 & $0.647 \pm 0.443$ & $1.016 \pm 0.468$ & $0.999 \pm 0.402$ \\
\hline \multicolumn{5}{|l|}{ Tstage } \\
\hline $\mathrm{T} 3+\mathrm{T} 4$ & 9 & $0.476 \pm 0.296$ & $1.306 \pm 0.324$ & $1.312 \pm 0.340$ \\
\hline $\mathrm{T} 1+\mathrm{T} 2$ & 30 & $0.751 \pm 0.426^{*}$ & $0.953 \pm 0.414^{*}$ & $0.911 \pm 0.303^{* *}$ \\
\hline \multicolumn{5}{|l|}{ Clinic stage } \\
\hline$I I I+I V$ & 23 & $0.466 \pm 0.283$ & $1.237 \pm 0.341$ & $1.199 \pm 0.304$ \\
\hline $\mid+\|$ & 16 & $1.006 \pm 0.362^{* *}$ & $0.743 \pm 0.347^{* *}$ & $0.722 \pm 0.187^{* *}$ \\
\hline \multicolumn{5}{|c|}{ Lymph node metastasis } \\
\hline Yes & 28 & $0.539 \pm 0.324$ & $1.171 \pm 0.316$ & $1.099 \pm 0.284$ \\
\hline No & 11 & $1.067 \pm 0.381^{* *}$ & $0.687 \pm 0.461^{* *}$ & $0.761 \pm 0.403^{*}$ \\
\hline
\end{tabular}

Data was shown as mean \pm SD. miR-324-3p, WNT2B mRNA and protein were normalized with the internal control U6, GAPDH and $\beta$-Actin, respectively * $P<0.05$

** $P<0.01$

mesenchymal marker Vimentin was considered as the classical indicators [27]. And EMT had been widely investigated to be classical mechanism of tumour invasion and metastasis [27, 28]. Moreover, recent researchers reported that miR-544a could induce EMT through the activation of WNT signaling pathway in gastric cancer [29]. In other tumours such as ovarian [30], colorectal [31] and tongue cancer [32], EMT was also associated with the activation of the WNT signalling pathway. With regard to NPC, we found that WNT2B was able to change the expression of E-cadherin and Vimentin [14]. In this study, we validated that miR-324-3p also had the ability of altering the expression of EMT biomarkers, which showed that miR-324-3p might regulate the migration and invasion of NPC through EMT.
In summary, we have demonstrated that miR-324-3p can target $W N T 2 B$ to regulate migration and invasion in NPC, and both miR-324-3p and target gene WNT2B were associated with $\mathrm{T}$ stage, clinic stage and cervical lymph node metastasis. Therefore, miR-324-3p/WNT2B axis may be potential therapeutic target for the treatment of patients with NPC.

\section{Conclusion}

miR-324-3p is a tumor-suppressive miRNA in NPC and inhibit NPC cell migration and invasion by targeting WNT2B. Complete understanding of the miR-324$3 \mathrm{p} / W N T 2 B$ pathway might contribute to discover new potential therapeutic clues for NPC.

\section{Additional files}

Additional file 1. qRT-PCR technical documentation

Additional file 2: Figure S1. Representative data of standard and melt curves of the premirs. (A) Standard curves of GAPDH and WNT2B. (B) Melt curves of GAPDH. (C) Melt curves of WNT2B. (D) Standard curves of U6 and miR-324-3p. (E) Melt curves of U6. (F) Melt curves of miR-324-3p. Figure S2. Validation of the stability of the reference genes. (A) Amplification plot and CT values of U6 between NPC and NPE. (B) Amplification plot and CT values of GAPDH between NPC and NPE. Figure S3. Spearman correlation analysis between the expression of miR-324-3p and WNT2B. (A) miR324-3p was negative correlated with WNT2B mRNA. (B) miR-324-3p was negative correlated with WNT2B protein. Figure S4. The expression data of NPE and NPC with two differential stratifications of T and clinic stage forms. (A) The expression of miR-324-3p between NPE and NPC. (B) The expression of WNT2B mRNA between NPE and NPC. (C) The expression of WNT2B mRNA between NPE and NPC.

\section{Abbreviations}

NPC: nasopharyngeal carcinoma; NPE: nasopharyngeal epithelium; NC: negative control; EMT: epithelial-mesenchymal transition; UTR: untranslated regions; qRT-PCR: quantitative real time PCR.

\section{Authors' contributions}

$L C, L Y$ and $Q Y Z$ conceived and designed the experiments. $L C, L Y, Q Y Z, L G$ and YNT performed the experiments. LC, SZW, ZST and DTB analyzed the data. RSL, LSH and TYQ contributed reagents and tissues collection. LC and LY wrote the paper. All authors read and approved the final manuscript. 


\author{
Author details \\ ${ }^{1}$ Department of Otolaryngology Head and Neck Surgery, Xiangya Hospital, \\ Central South University, 87 Xiangya Road, Changsha 410008, Hunan, China. \\ ${ }^{2}$ Otolaryngology Major Disease Research Key Laboratory of Hunan Province, \\ Changsha 410008, Hunan, China.
}

\section{Acknowledgements}

None.

\section{Competing interests}

The authors declare that they have no competing interests.

\section{Availability of data and materials}

The datasets supporting the conclusions of this article are included within the article.

\section{Ethics approval and consent to participate}

This research was reviewed and approved by the Ethic Committee of Xiangya Hospital, Central South University (Study ID number 201303151). Informed consent was obtained from all individual participants included in the study.

\section{Funding}

Grants were provided by the National Natural Science Foundation of China (No. 81372426), the National Natural Science Foundation of Hunan Province (No. 2015JJ3137), the Youth Fund of Xiangya Hospital (No. 2015Q04) and the Open Fund of Key Laboratory of Molecular Radiation Oncology, Hunan Province.

Received: 12 September 2016 Accepted: 15 December 2016 Published online: 03 January 2017

\section{References}

1. Torre LA, Bray F, Siegel RL, Ferlay J, Lortet-Tieulent J, Jemal A. Global cancer statistics 2012. CA Cancer J Clin. 2015;65:87-108.

2. Razak AR, Siu LL, Liu FF, Ito E, O'Sullivan B, Chan K. Nasopharyngeal carcinoma: the next challenges. Eur J Cancer. 2010;46:1967-78.

3. Bensouda Y, Kaikani W, Ahbeddou N, Rahhali R, Jabri M, Mrabti H, Boussen $\mathrm{H}$, Errihani $\mathrm{H}$. Treatment for metastatic nasopharyngeal carcinoma. Eur Ann Otorhinolaryngol Head Neck Dis. 2011;128:79-85.

4. Bartel DP. MicroRNAs: genomics, biogenesis, mechanism, and function. Cell. 2004;116:281-97.

5. Lytle JR, Yario TA, Steitz JA. Target mRNAs are repressed as efficiently by microRNA-binding sites in the $5^{\prime}$ UTR as in the $3^{\prime}$ UTR. Proc Natl Acad Sci USA. 2007:104:9667-72.

6. Gu W, Wang X, Zhai C, Zhou T, Xie X. Biological basis of miRNA action when their targets are located in human protein coding region. PLOS ONE. 2013;8:e63403.

7. Di Leva G, Garofalo M, Croce CM. MicroRNAs in cancer. Annu Rev Pathol. 2014;9:287-314

8. Ou H, Li Y, Kang M. Activation of miR-21 by STAT3 induces proliferation and suppresses apoptosis in nasopharyngeal carcinoma by targeting PTEN gene. PLOS ONE. 2014;9:e109929.

9. Qu JQ, Yi HM, Ye X, Li LN, Zhu JF, Xiao T, Yuan L, Li JY, Wang YY, Feng J, He QY, Lu SS, Yi H, Xiao ZQ. MiR-23a sensitizes nasopharyngeal carcinoma to irradiation by targeting IL-8/Stat3 pathway. Oncotarget. 2015;6:28341-56.

10. Peng X, Cao P, Li J, He D, Han S, Zhou J, Tan G, Li W, Yu F, Yu J, Li Z, Cao K. MiR-1204 sensitizes nasopharyngeal carcinoma cells to paclitaxel both in vitro and in vivo. Cancer Biol Ther. 2015;16:261-7.

11. Huang H, Sun P, Lei Z, Li M, Wang Y, Zhang HT, Liu J. MiR-145 inhibits invasion and metastasis by directly targeting Smad3 in nasopharyngeal cancer. Tumour Biol. 2015;36:4123-31.

12. Fang Y, Zhu X, Wang J, Li N, Li D, Sakib N, Sha Z, Song W. MiR-744 func tions as a proto-oncogene in nasopharyngeal carcinoma progression and metastasis via transcriptional control of ARHGAP5. Oncotarget. 2015;6:13164-75.
13. Li G, Liu Y, Su Z, Ren S, Zhu G, Tian Y, Qiu Y. MicroRNA-324-3p regulates nasopharyngeal carcinoma radioresistance by directly targeting WNT2B. Eur J Cancer. 2013;49:2596-607.

14. Li G, Wang Y, Liu Y, Su Z, Liu C, Ren S, Deng T, Huang D, Tian Y, Qiu Y. MiR185-3p regulates nasopharyngeal carcinoma radioresistance by targeting WNT2B in vitro. Cancer Sci. 2014;105:1560-8.

15. Kim J, Krichevsky A, Grad Y, Hayes GD, Kosik KS, Church GM, Ruvkun G. Identification of many microRNAs that copurify with polyribosomes in mammalian neurons. Proc Natl Acad Sci USA. 2004;101:360-5.

16. Hu Z, Dong J, Wang LE, Ma H, Liu J, Zhao Y, Tang J, Chen X, Dai J, Wei Q, Zhang C, Shen $\mathrm{H}$. Serum microRNA profiling and breast cancer risk: the use of miR-484/191 as endogenous controls. Carcinogenesis. 2012;33:828-34.

17. Wen Y, Han J, Chen J, Dong J, Xia Y, Liu J, Jiang Y, Dai J, Lu J, Jin G, Han J, Wei Q, Shen H, Sun B, Hu Z. Plasma miRNAs as early biomarkers for detecting hepatocellular carcinoma. Int J Cancer. 2015;137:1679-90.

18. Namkung J, Kwon W, Choi Y, Yi SG, Han S, Kang MJ, Kim SW, ParkT, Jang JY. Molecular subtypes of pancreatic cancer based on miRNA expression profiles have independent prognostic value. J Gastroenterol Hepatol. 2016:31:1160-7.

19. Macconi D, Tomasoni S, Romagnani P, Trionfini P, Sangalli F, Mazzinghi $B$, Rizzo P, Lazzeri E, Abbate M, Remuzzi G, Benigni A. MicroRNA-324-3p promotes renal fibrosis and is a target of ACE inhibition. J Am Soc Nephrol. 2012;23:1496-505.

20. Dharap A, Pokrzywa C, Murali S, Pandi G, Vemuganti R. MicroRNA miR324-3p induces promoter-mediated expression of RelA gene. PLOS ONE. 2013;8:e79467.

21. Xu J, Ai Q, Cao H, Liu Q. MiR-185-3p and miR-324-3p predict radiosensitivity of nasopharyngeal carcinoma and modulate cancer cell growth and apoptosis by targeting SMAD7. Med Sci Monit. 2015;21:2828-36.

22. Katoh M. Differential regulation of WNT2 and WNT2B expression in human cancer. Int J Mol Med. 2001:8:657-60.

23. Li SJ, Yang XN, Qian HY. Antitumor effects of WNT2B silencing in GLUT1 overexpressing cisplatin resistant head and neck squamous cell carcinoma. Am J Cancer Res. 2015;5:300-8.

24. Kobayashi M, Huang CL, Sonobe M, Kikuchi R, Ishikawa M, Kitamura J, Miyahara R, Menju T, Iwakiri S, Itoi K, Yasumizu R, Date H. Intratumoral Wnt2B expression affects tumor proliferation and survival in malignant pleural mesothelioma patients. Exp Ther Med. 2012;3:952-8.

25. Jiang H, Li F, He C, Wang X, Li Q, Gao H. Expression of Gli1 and Wnt2B correlates with progression and clinical outcome of pancreatic cancer. Int J Clin Exp Pathol. 2014;7:4531-8.

26. Wang H, Fan L, Xia X, Rao Y, Ma Q, Yang J, Lu Y, Wang C, Ma D, Huang X. Silencing Wnt2B by siRNA interference inhibits metastasis and enhances chemotherapy sensitivity in ovarian cancer. Int J Gynecol Cancer. 2012;22:755-61.

27. Iwatsuki M, Mimori K, Yokobori T, Ishi H, Beppu T, Nakamori S, Baba H, Mori M. Epithelial-mesenchymal transition in cancer development and its clinical significance. Cancer Sci. 2010;101:293-9.

28. Guarino M, Rubino B, Ballabio G. The role of epithelial-mesenchymal transition in cancer pathology. Pathology. 2007;39:305-18.

29. Yanaka Y, Muramatsu T, Uetake H, Kozaki K, Inazawa J. MiR-544a induces epithelial-mesenchymal transition through the activation of WNT signaling pathway in gastric cancer. Carcinogenesis. 2015;36:1363-71.

30. Baldwin LA, Hoff JT, Lefringhouse J, Zhang M, Jia C, Liu Z, Erfani S, Jin H, Xu M, She QB, van Nagell JR, Wang C, Chen L, Plattner R, Kaetzel DM, Luo J, Lu M, West D, Liu C, Ueland FR, Drapkin R, Zhou BP, Yang XH. CD151alpha3beta1 integrin complexes suppress ovarian tumor growth by repressing slug-mediated EMT and canonical Wnt signaling. Oncotarget. 2014;5:12203-17.

31. Vincan E, Barker N. The upstream components of the Wnt signalling pathway in the dynamic EMT and MET associated with colorectal cancer progression. Clin Exp Metastasis. 2008;25:657-63.

32. Liang J, Liang L, Ouyang K, Li Z, Yi X. MALAT1 induces tongue cancer cells' EMT and inhibits apoptosis through Wnt/beta-catenin signaling pathway. J Oral Pathol Med. 2016. doi:10.1111/jop.12466. 